\title{
Effect of caffeine on the multiplication of DNA and RNA viruses
}

\author{
MASAKI MURAYAMA $^{1}$, KAZUKO TSUJIMOTO ${ }^{1,2}$, MISAO UOZAKI ${ }^{1}$, YUKIKO KATSUYAMA ${ }^{1}$, \\ HISASHI YAMASAKI ${ }^{1}$, HIROTOSHI UTSUNOMIYA ${ }^{2}$ and A. HAJIME KOYAMA ${ }^{1}$ \\ ${ }^{1}$ Division of Virology, Department of Cellular and Molecular Medicine, and ${ }^{2}$ Department of Strategic Surveillance for \\ Functional Food and Comprehensive Traditional Medicine, Wakayama Medical University \\ Graduate School of Medicine, Wakayama 641-8509, Japan
}

Received December 3, 2007; Accepted January 8, 2008

\begin{abstract}
The present study examined the effect of caffeine on RNA and DNA viruses, revealing that it inhibits the multiplication of both. In the presence of caffeine, the progeny virus yield of both herpes simplex virus type 1 (HSV-1) and poliovirus decreased with increasing concentrations of the reagent, although HSV-1 was much more sensitive than poliovirus. The influenza virus was not affected by caffeine at the same concentrations. None of the viruses were directly inactivated by caffeine at the tested concentrations. Characterization of the mode of action of caffeine against HSV-1 infection revealed that the addition of the reagent at $10 \mathrm{~h}$ post-infection significantly affected the formation of progeny virus, indicating that caffeine can inhibit the multiplication of HSV-1 during the step(s) following the completion of viral DNA replication and the formation of nucleocapsids. In addition, the reagent selectively enhanced the cytopathic effects and cell death of the infected cells over uninfected cells, suggesting that the antiviral action of caffeine against $\mathrm{HSV}-1$ is, at least in part, the result of accelerated degeneration of the infected cells.
\end{abstract}

\section{Introduction}

Previously, we showed that hot water extracts of coffee grinds and commercial instant coffee solutions exhibited marked antiviral activities against herpes simplex virus type 1 (HSV-1) and poliovirus (unpublished data). Among the components of coffee extracts, caffeine has been reported to show antiviral activity against HSV-1 $(1,2)$, although we found that caffeine was not the major antiviral component of the coffee extracts.

Correspondence to: Dr A. Hajime Koyama, Division of Virology, Department of Cellular and Molecular Medicine, Wakayama Medical University Graduate School of Medicine, 811-1 Kimiidera, Wakayama 641-8509, Japan

E-mail:koyama@wakayama-med.ac.jp

Key words: caffeine, antiviral, herpes simplex virus, influenza virus, poliovirus, apoptosis
Caffeine is a methyl-xanthine alkaloid compound that is attracting attention due to the variety of physiological and pharmacological activities it possesses $(3,4)$. In terms of its antiviral activity, Yamazaki and Tagaya (1) reported their preliminary observations of the antiviral activities of caffeine in certain viruses, such as poliovirus, influenza virus, HSV-1 and vaccinia virus, but not in Newcastle disease virus (NDV), Japanese encephalitis virus or adenovirus. However, their results were not fully consistent with subsequent studies; for example, Olson and Consigli (7) found that caffeine showed antiviral activities against NDV by inhibiting the synthesis of viral RNA and proteins in the infected cells. At present, the consensus appears to be that caffeine inhibits the growth of NDV, human immunodeficiency virus, polyomavirus, HSV-1 and vaccinia virus $(1,2,5-7)$. We therefore reexamined the antiviral effects of caffeine on viruses of different classes, namely poliovirus, influenza virus and HSV-1.

Regarding the effect of caffeine on the multiplication of HSV-1, Shiraki and Rapp (2) reported that caffeine inhibited viral protein synthesis and suppressed the plaque formation of HSV-1 by interfering with the cell-to-cell transmission of the virus. However, they only characterized viral multiplication for up to $8 \mathrm{~h}$ post-infection in most of their experiments, almost midway to the completion of normal HSV-1 replication ( $\sim 14 \mathrm{~h})$. During the quantitative characterization of virus-host interaction in the HSV-1-infected cells, we noticed that caffeine preferentially accelerated the cytopathic effects (CPE) and death of infected cells. A similar observation was made with viral infection in the presence of tumor necrosis factor (8) or gallate derivatives (9). Such preferential CPE on infected cells should result in reduced virus yields, and hence raises the possibility of new types of antiviral drugs that could induce the selective death of virus-infected cells. In view of the possibility that caffeine may belong to this new class of antiviral agents, we further characterized its antiviral actions.

\section{Materials and methods}

Cells and viruses. MDCK, HEp-2 and Vero cells were grown in Eagle's minimum essential medium (MEM) containing 10\% newborn calf serum. Herpes simplex virus type 1, strain F, (HSV-1), influenza virus A/Aichi/68 $\left(\mathrm{H}_{3} \mathrm{~N}_{2}\right)$ and poliovirus type 1 , Sabin vaccine strain, were used as test viruses. The influenza virus was propagated in MDCK cells in MEM 
supplemented with $0.1 \%$ bovine serum albumin (BSA) and acetylated trypsin $(4 \mu \mathrm{g} / \mathrm{ml})$, while both $\mathrm{HSV}-1$ and poliovirus were propagated in Vero cells in MEM supplemented with $0.5 \%$ fetal bovine serum. These viruses were stored at $-80^{\circ} \mathrm{C}$ until use. The amount of each virus was measured by a plaque assay as described previously (10-12).

Virus yields in the presence of caffeine. Caffeine was purchased from Wako Chemical. A stock solution $(100 \mathrm{mM})$ was prepared by dissolving the reagent in hot Dulbecco's phosphatebuffered saline without $\mathrm{Ca}^{++}$and $\mathrm{Mg}^{++}$(PBS), followed by filtration through a Millipore Millex-GV membrane (pore size, $0.22 \mu \mathrm{m}$ ). This was then stored in a refrigerator until use.

Monolayered cells in 35-mm dishes were infected with the virus at an indicated multiplicity of infection (MOI). The infected cells were further incubated at $37^{\circ} \mathrm{C}$ (for $\mathrm{HSV}-1$ and influenza virus) or $35.5^{\circ} \mathrm{C}$ (for poliovirus) for the indicated period in the serum-free MEM containing 0.1\% BSA and the indicated concentrations of caffeine. In the case of the influenza virus, acetylated trypsin $(4 \mu \mathrm{g} / \mathrm{ml})$ was also added to the medium for the activation of virus infectivity. At the end of infection with influenza virus, the culture medium was harvested and the amount of infectious virus in the medium was determined as a total progeny virus. For HSV-1 and poliovirus, the amount of total progeny virus in the infected culture was determined at the indicated time after the infected cells, along with the culture media, had undergone two or three cycles of freezing and thawing.

Assay for virucidal activity. All the starting materials were stored on ice prior to the virus inactivation experiments. A $475-\mu 1$ aliquot of the solutions to be tested was placed in a $1.5-\mathrm{ml}$ plastic tube on ice and received $25 \mu 1$ of virus preparations $\left[\sim 10^{8}\right.$ or $10^{9}$ plaque-forming units $\left.(\mathrm{PFU}) / \mathrm{ml}\right]$. This was immediately followed by vigorous mixing, then incubation on ice. At the end of incubation, aliquots of the virus samples were diluted 100-fold with ice-cold PBS containing $1 \%$ calf serum (HSV-1 or poliovirus) or $0.1 \%$ BSA (influenza virus). The viruses were further diluted to determine the number of infectious viruses in the treated preparation by plaque assay. All the experiments were conducted in duplicate or triplicate (13).

Determination of CPE and cell death. Monolayers of Vero cells were mock-infected or infected with HSV-1 at the indicated MOI. The infected cells were incubated in the serum-free MEM containing $0.1 \% \mathrm{BSA}$ and the indicated concentrations of caffeine. The CPE were determined by microscopic observation of the cells; approximate amounts of rounded cells on monolayers were estimated under a phase contrast microscope. In the case of the influenza virus infection, MDCK cells and the serum-free MEM containing $0.1 \% \mathrm{BSA}$ and acetylated trypsin $(4 \mu \mathrm{g} / \mathrm{ml})$ were used in the same manner as in HSV-1.

To determine the extent of cell death in the cultures, monolayered cells were trypsinized at the indicated time postinfection to obtain a single-cell suspension. After the addition of MEM containing $10 \%$ calf serum to the suspension to neutralize the trypsin and stabilize the cells, the number of living and dead cells was determined by a dye-exclusion method with trypan blue.

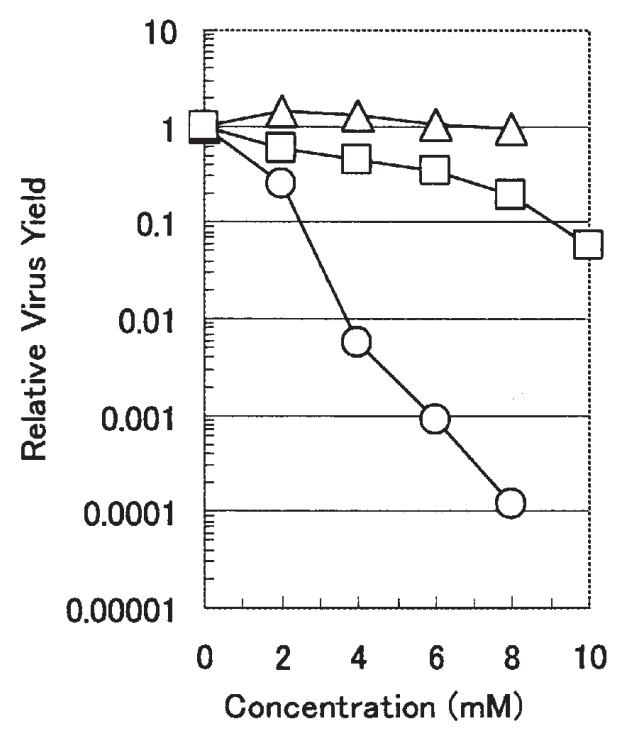

Figure 1. Effect of caffeine on virus yields. Confluent monolayers of HEp-2 (for HSV-1 and poliovirus) or MDCK (for influenza virus) cells were infected with each of the three viruses at an MOI of 10 for HSV-1, 9 for influenza virus and 5 for poliovirus. The infected cells were incubated overnight in medium containing varying concentrations of caffeine at $37^{\circ} \mathrm{C}$ for $\mathrm{HSV}-1$ and influenza virus or $35.5^{\circ} \mathrm{C}$ for poliovirus. At the end of infection, the amounts of infectious progeny virus were determined and normalized to the virus yield in the absence of the reagent. $\odot$, HSV $-1 ; \triangle$, influenza virus; $\square$, poliovirus.

Observation of cell nuclei. The cells, grown on cover glasses in dishes, were mock-infected or infected with a respective virus and were incubated in an appropriate medium. At various times following infection, the infected cells on the glass were fixed with acetic acid and methanol (1:3) and the cell nuclei were stained with DNA-binding dye Hoechst 33258 (14).

\section{Results and Discussion}

Effect of caffeine on the multiplication of viruses. To examine the antiviral activities of caffeine, we tested three viruses of completely different types: HSV-1 (Herpesviridae family), influenza virus (Orthomyxoviridae family) and poliovirus (Picornaviridae family). Both HSV-1 (DNA virus) and influenza virus (RNA virus) are large enveloped viruses and need a cell nucleus for virus multiplication $(15,16)$, while poliovirus (RNA virus) is a small non-enveloped virus and replicates in the cytoplasm of the infected cells (17).

Fig. 1 shows the effects of caffeine on the relative virus yields of these three viruses, when the infected cells were incubated in medium containing the indicated concentrations of the reagent. The multiplication of HSV-1 was highly sensitive to the reagent; the virus yield decreased as the reagent concentration was increased to $8 \mathrm{mM}$ and, in the presence of $4 \mathrm{mM}$ caffeine, the yield decreased $>100$-fold. Two RNA viruses, influenza virus and poliovirus, were not as sensitive to the reagent as HSV-1. The multiplication of influenza virus was apparently not affected by the reagent at these concentrations, while poliovirus was slightly, but significantly, affected by caffeine at higher concentrations; for example, the progeny virus yields of poliovirus decreased $\sim 10$-fold at $10 \mathrm{mM}$ of caffeine. These results show that caffeine inhibits the multiplication of both DNA and RNA viruses, which widely differ in virion structure (enveloped or non-enveloped) and in the 
way genome replication and transcription occur (in the nucleus or in the cytoplasm of infected cells). This is in agreement with the previous preliminary report of Yamazaki and Tagaya (1). However, in contrast to their report, caffeine was not effective against the influenza virus (Fig. 1), indicating that not all viruses are sensitive to the reagent. The discrepancy between their results and ours may be due to the difference in the virus strain (H1N1 vs. H3N2) or cell type (CE vs. MDCK) used.

Previously, we found that coffee extracts exert not only antiviral activity, but also virucidal activity, against HSV-1. Therefore, the three viruses were incubated on ice for $60 \mathrm{~min}$ with the reagent at different concentrations, and the virucidal activity of caffeine was examined. As shown in Fig. 2, caffeine showed no apparent virucidal activity; infectivity of HSV-1, influenza virus and poliovirus was not affected by it, even at $20 \mathrm{mM}$. This suggests that the observed virucidal activity of coffee extracts is due to a component (or components) other than caffeine, which may be consistent with the previous observation that caffeine is not a major part of the antiviral activities of coffee extracts.

Multiplication of HSV-1 in the presence of caffeine. Since HSV-1 was the virus most sensitive to caffeine, experiencing a unique mode of degeneration of the infected cells (see below), we further characterized the mode of HSV-1 infection in the presence of caffeine. Fig. 3 shows one-step growth curves of HSV-1 in HEp-2 cells in the presence or absence of caffeine. In the absence of the reagent, the progeny infectious virus appeared after $6 \mathrm{~h}$ post-infection (h p.i.) and rapidly increased for the following $6 \mathrm{~h}$, leveling off to a plateau at $\sim 14 \mathrm{~h} \mathrm{p.i.}$ However, in the presence of caffeine at $4 \mathrm{mM}$, the multiplication of the virus was extensively suppressed. The formation of infectious progeny virus appears to have started at $6 \mathrm{~h}$ p.i., but with a rate notably suppressed. The final virus yield was 100 -fold less than the yield in the absence of the reagent.

Previously, we quantitatively characterized the kinetics of viral DNA replication, the encapsidation of viral DNA, the envelopment of nucleocapsids and the formation of infectious progeny virus (18) and revealed that viral DNA replication occurs exclusively between $3 \mathrm{~h}$ p.i. and $6 \mathrm{~h}$ p.i. in HSV-1infected cells. To examine the target of the anti-herpetic activity of caffeine, the reagent was added to the infected culture at $10 \mathrm{~h}$ p.i., when the replication of virus DNA was completed and a large number of nucleocapsids had accumulated in the infected cells (18). As shown in Fig. 3, the amount of progeny virus (black squares) continued to increase for an additional $2 \mathrm{~h}$ after the addition of caffeine, then leveled off at a significantly reduced progeny virus yield. These results clearly show that the reagent can interfere with virus multiplication after viral DNA replication, probably at the formation of progeny infectious virus step, because similar kinetics were observed when the multiplication of HSV-1 was inhibited by ammonium chloride (19) or Brefeldin A (10) during the formation of infectious progeny virus after viral DNA replication and nucleocapsid formation.

Effect of caffeine on the infected cells. Previously, we observed that the suppression of progeny virus production is often accompanied by a significant acceleration of virus-infected cell death $(8,9$, reviewed in ref. 20$)$. In agreement with these

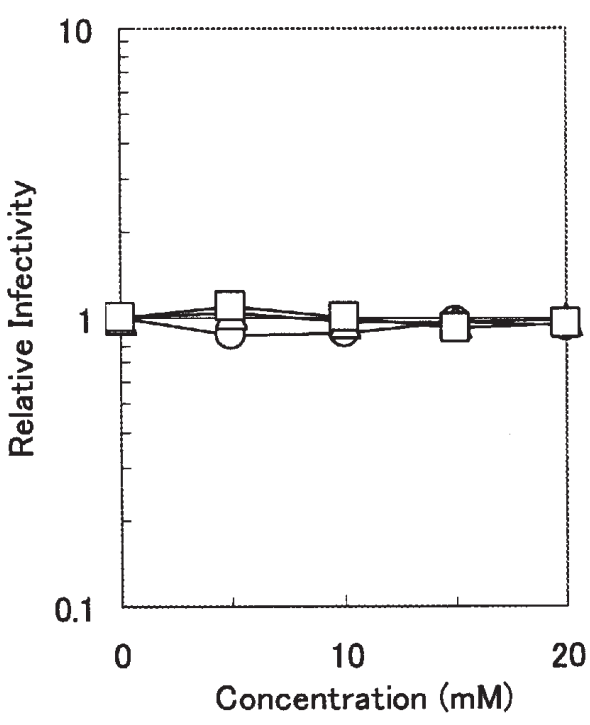

Figure 2. Direct virucidal effect of caffeine. Aliquots of virus preparation were incubated on ice for $60 \mathrm{~min}$ in the presence of various concentrations of caffeine. The number of infectious viruses after incubation was determined by the plaque method. $\odot$, HSV-1; $\triangle$, influenza virus; $\square$, poliovirus.

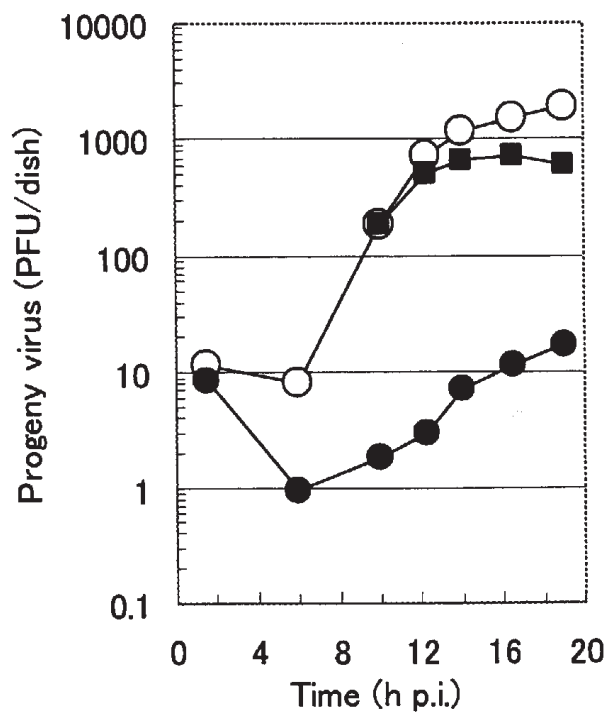

Figure 3. One-step growth curve of HSV-1 in the presence or absence of caffeine. Confluent monolayers of HEp-2 cells were infected with HSV-1 at an MOI of 8 . The infected cells were incubated at $37^{\circ} \mathrm{C}$ in MEM containing $0.1 \%$ BSA $(\bigcirc)$ or in medium additionally containing $4 \mathrm{mM}$ caffeine $(\bullet)$. Some of the dishes with the infected cells received the same concentration of caffeine at $10 \mathrm{~h}$ p.i. ( $\square$ ). At the indicated times, the amount of infectious progeny virus was determined after the infected cells, along with the culture medium, had undergone three cycles of freezing and thawing.

observations, the HSV-1-infected cells showed marked CPE (cell-rounding and detachment from the dish surface) in the presence of caffeine compared to in its absence, although HSV-1 usually does not induce significant CPE in infected HEp-2 cells until very late in the infection.

Fig. 4 shows the kinetics of the induction of death of the infected cells (A) and of the production of cell-free virus during the course of infection with HSV-1 (B). The production of cell-free virus (the virus population released from the 

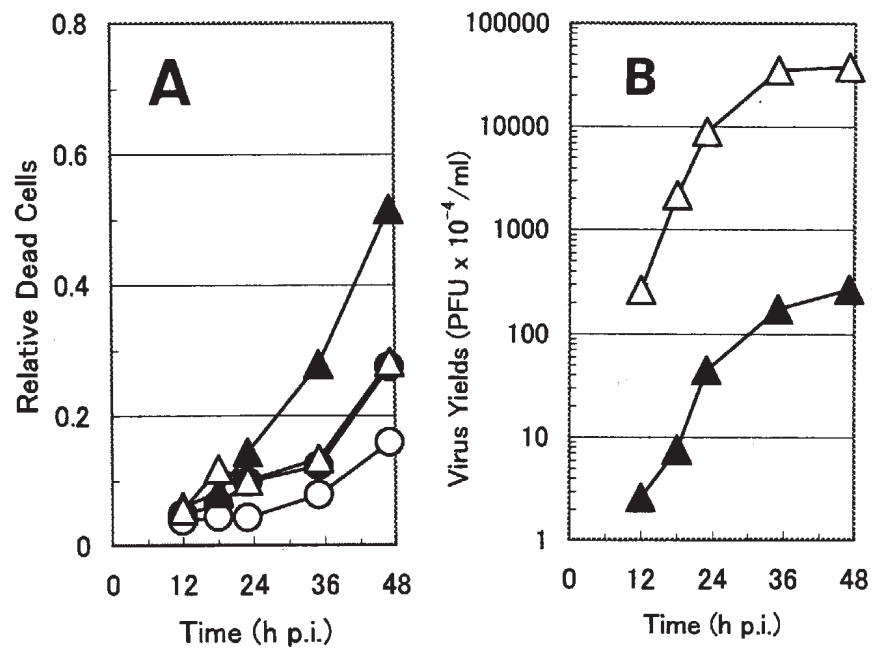

Figure 4. Confluent monolayers of HEp-2 cells mock-infected $(\circ, \bullet)$ or infected with HSV-1 $(\triangle, \boldsymbol{\Delta})$ at an MOI of 14 . The infected cells were incubated in medium supplemented with $(\boldsymbol{\bullet}, \mathbf{\Delta})$ or without $(\bigcirc, \triangle) 5 \mathrm{mM}$ caffeine at $37^{\circ} \mathrm{C}$. (A) At the indicated times, the number of living and dead cells was determined as described in Materials and methods. (B) At the indicated times, the amount of progeny infectious virus in the medium (the cell-free virus) was determined.
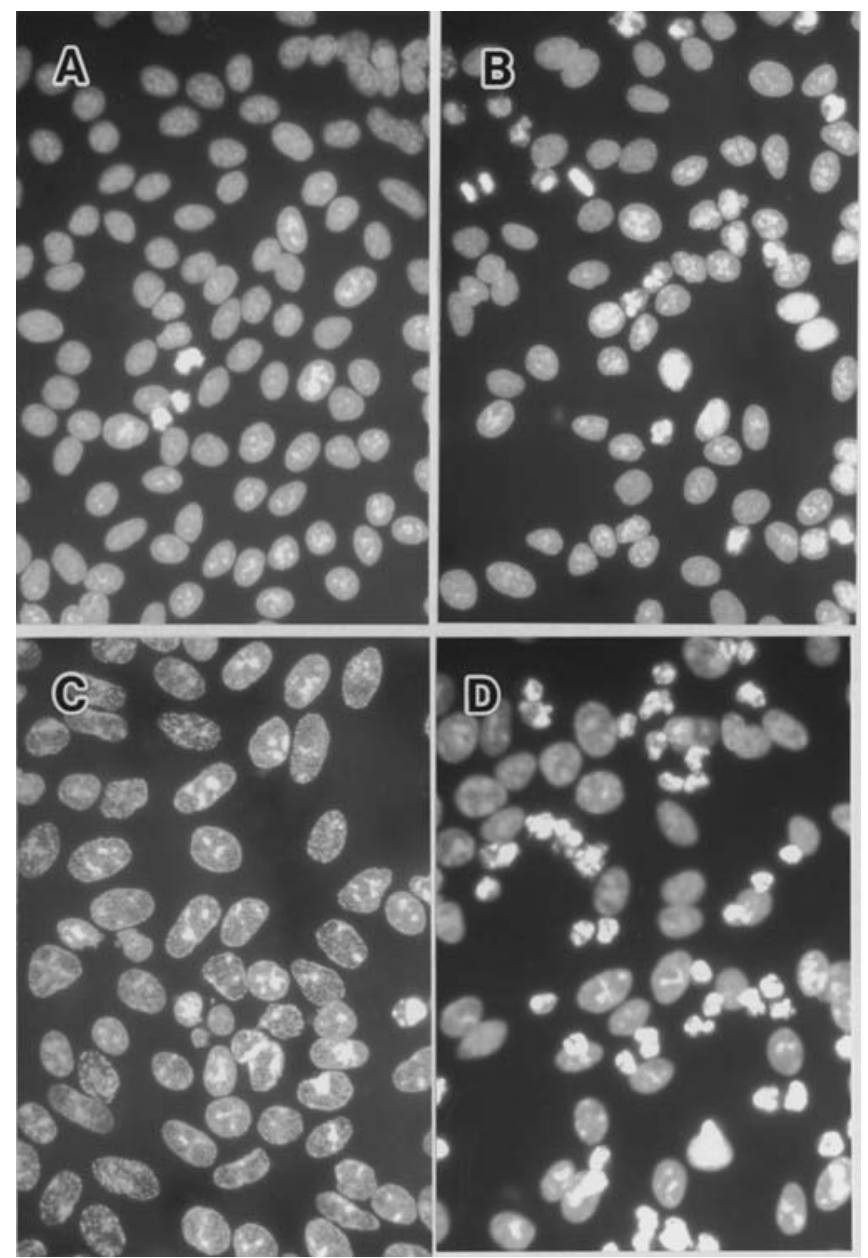

Figure 5. Effect of caffeine on the nuclear morphology of infected cells. Confluent monolayers of HEp-2 cells were mock-infected (A and B) or infected with HSV-1 at an MOI of 10 (C and D). The infected cells were incubated at $37^{\circ} \mathrm{C}$ in medium containing $4 \mathrm{mM}$ caffeine (B and D) or not (A and $\mathrm{C})$. At $14 \mathrm{~h}$ p.i., the infected cells were fixed and stained with Hoechst 33258 . infected cells) follows the production of intracellular infectious progeny virus but is known to show a lag period, especially at a later stage of infection. Under our experimental conditions, the increase in the amount of cell-free virus started before $12 \mathrm{~h}$ p.i., increased with time and reached a plateau at $\sim 36 \mathrm{~h}$ p.i. in both the reagent-treated and untreated cultures (Fig. 4B). When the kinetics of the induction of cell death were compared in the mock- and HSV-1-infected cells in the presence or absence of caffeine (Fig. 4A), the virus-infected cells in the presence of the reagent showed the highest rate of cell death. Note that $4 \mathrm{mM}$ of caffeine alone (i.e., without virus infection) somehow enhanced the rate of cell death during incubation, indicating the significant cytotoxicity of the reagent. The difference in cell death was most significant at $36 \mathrm{~h}$ p.i., when the production of cell-free virus reached a maximum. These results support the notion that, in the case of HSV-1 infection, the antiviral activity of caffeine results from its selective degeneration of virus-infected over uninfected cells.

However, it should be noted that in the case of influenza virus and poliovirus the acceleration of both CPE and cell death by caffeine was not detected (data not shown). This is probably because these two viruses induced massive CPE and cell death of the infected cells rapidly after infection, which might have masked the acceleration of CPE and cell death, if any occured. The suppression of virus-induced CPE was even observed in the case of poliovirus infection, suggesting that the caffeine-induced selective degeneration of the infected cells may be dependent on the species of virus.

To confirm and further characterize the selective cytotoxicity of caffeine in HSV-1-infected cells, we examined the morphological changes of the infected cell nuclei. While mockinfected cells showed relatively small nuclei with homogeneous morphology in the absence of caffeine (Fig. 5A), the virus-infected cells showed enlarged nuclei (Fig. 5C). In the presence of the reagent, the HSV-1-infected cells showed a considerable number of cell nuclei with condensation and fragmentation of chromatin (Fig. 5D), a classic sign of apoptotic cell nuclei, while the mock-infected cells (Fig. 5B) essentially showed a similar morphology to that exhibited in the absence of reagent (Fig. 5A). The presence of a few apoptotic nuclei in Fig. 5B is in agreement with the observed cytotoxicity of the reagent (Fig. 4A). From these observations, we propose that caffeine inhibits virus multiplication at least in part as a result of the acceleration of the selective degeneration (apoptosis) of virus-infected cells.

In conclusion, we have shown that the multiplication of HSV-1 and poliovirus is sensitive to caffeine, indicating that caffeine can inhibit both DNA and RNA viruses. However, the multiplication of influenza virus in vitro was not sensitive to the reagent, indicating that not all viruses are sensitive to caffeine. Caffeine did not inactivate any of the three viruses but did inhibit the multiplication of HSV-1, most likely at the final step of infectious progeny virus formation, although its effects on some other steps of the virus multiplication process cannot be excluded. Additionally, like TNF or octyl gallate, caffeine induces selective apoptosis in virus-infected cells. This last finding may initiate a new strategy for the discovery of novel antiviral drugs that inhibit viral growth by accelerating cellular response against virus infection to induce apoptosis and abort infection. 


\section{Acknowledgements}

The authors thank Dr Tsutomu Arakawa (Alliance Protein Lab, CA) for his stimulating discussions and advice. This work was supported in part by a research grant from All Japan Coffee Association.

\section{References}

1. Yamazaki $\mathrm{Z}$ and Tagaya I: Antiviral effect of atropine and caffeine. J Gen Virol 50: 429-431, 1980.

2. Shiraki K and Rapp F: Effects of caffeine on herpes simplex virus. Intervirology 29: 235-240, 1988.

3. Cowan MM: Plant products as antimicrobial agents. Clin Microbiol Rev 12: 564-582, 1999.

4. Daly JW: Caffeine analogs: biomedical impacts. Cell Mol Life Sci 64: 2153-2169, 2007.

5. Daniel R, Marusich E, Argyris E, Zhao RY, Skalka AM and Pomerantz RJ: Caffeine inhibits human immunodeficiency virus type 1 transduction of nondividing cells. J Virol 76: 2058-2065, 2005.

6. Dahl J, You J and Benjamin TL: Induction and utilization of an ATM signaling pathway by polyomavirus. J Virol 79: 13007-13017, 2005.

7. Olson NJ and Consigli RA: Production of labile Newcastle disease virus progeny after infection of chicken embryo cells in the presence of caffeine. Am J Vet Res 40: 387-392, 1979.

8. Koyama AH, Arakawa T and Adachi A: Acceleration of virusinduced apoptosis by tumor necrosis factor. FEBS Lett 426 : 179-182, 1998.

9. Uozaki M, Yamasaki H, Katsuyama Y, Higuchi M, Higuchi T and Koyama AH: Antiviral effect of octyl gallate against DNA and RNA viruses. Antiviral Res 73: 85-91, 2007.
10. Koyama $\mathrm{AH}$ and Uchida $\mathrm{T}$ : Inhibition by Brefeldin $\mathrm{A}$ of the envelopment of nucleocapsids in herpes simplex virus type 1infected Vero cells. Arch Virol 135: 305-317, 1994.

11. Kurokawa M, Koyama AH, Yasuoka S and Adachi A: Influenza virus overcomes apoptosis by rapid multiplication. Int J Mol Med 3: 527-530, 1999.

12. Koyama AH, Irie H, Ueno F, Ogawa M, Nomoto A and Adachi A: Suppression of apoptotic and necrotic cell death by poliovirus. J Gen Virol 82: 2965-2972, 2001.

13. Yamasaki H, Tsujimoto K, Koyama AH and Arakawa T: Arginine facilitates virus inactivation. J Pharm Sci (In press).

14. McGarrity GJ: Detection of contamination. Methods Enzymol 58: 18-29, 1979.

15. Roizman B and Knipe DM: Herpes simplex virus and their replication. In: Fields Virology. 4th edition. Fields BN, Knipe DM and Howley PM (eds). Lippincott-Raven, New York, pp2399-2460, 2001.

16. Lamb RA and Krug RM: Orthomyxoviridae: The viruses and their replication. In: Fields Virology. 4th edition. Fields BN, Knipe DM and Howley PM (eds). Lippincott-Raven, New York, pp1487-1530, 2001.

17. Racaniello VR: Picornaviridae: The viruses and their replication. In: Fields Virology. 4th edition. Fields BN, Knipe DM and Howley PM (eds). Lippincott-Raven, New York, pp685-722, 2001.

18. Koyama AH and Uchida T: Quantitative studies on the maturation process of herpes simplex virus type 1 in Vero cells. Virus Res 10: 281-286, 1988.

19. Koyama AH and Uchida T: The effect of ammonium chloride on the multiplication of herpes simplex virus type 1 in Vero cells. Virus Res 13: 271-282, 1989.

20. Koyama AH, Fukumori T, Fujita M, Irie $\mathrm{H}$ and Adachi A: Physiological significance of apoptosis in animal virus infection. Microbes Infect 2: 1111-1117, 2000. 